\section{Dr. Genovese comments}

To the Editor:

The Abatacept Comparison of sub(QU)cutaneous versus intravenous in Inadequate Responders to methotrexatE (ACQUIRE) trial was a multinational, Phase IIIb, randomized, double-blind study that evaluated the comparable efficacy and safety of subcutaneous (SC) and intravenous (IV) abatacept (ABA) over 6 months ${ }^{1}$. At Month 6 , similar proportions of $\mathrm{SC}$ and IV ABA-treated patients achieved an American College of Rheumatology 20 response (estimated difference: $0.3 \%, 95 \% \mathrm{CI}-4.2,4.8$ ), confirming noninferiority of SC to IV ABA. The onset and magnitude of efficacy responses were equal for both formulations, and similar patient retention was also reported (94.2\% for SC ABA vs $93.8 \%$ for IV ABA at Month 6). Overall safety was also similar between groups, including discontinuations due to adverse events and serious adverse events, serious infections, malignancies, and autoimmune events. However, the trial did not look directly at the question of switching from IV to SC in the parent trial. That aspect was considered in the longterm extension (LTE) study ${ }^{2}$, in which all patients who completed the 6-month double-blind period received SC ABA $125 \mathrm{mg}$ weekly for up to $\sim 3.5$ years of exposure, to assess its longterm safety, efficacy, and tolerability. Clinical and functional benefits were maintained longterm during the ACQUIRE LTE study, regardless of whether patients received $\mathrm{SC} A \mathrm{ABA}$ throughout or switched from IV ABA to $\mathrm{SC} \mathrm{ABA}$ at the start of the LTE.

These observations support findings from the ATTUNE study, which evaluated safety and efficacy in patients who switched from IV to SC ABA. The results of 2 large studies, ATTUNE ${ }^{3}$ and the ACQUIRE LTE $^{2}$, reached different conclusions from those of Reggia, et $a l^{4}$. However, one needs to remain cautious regarding overinterpretation of clinical trials data, because
ACQUIRE and ATTUNE had fairly homogeneous patient populations followed in the context of fairly rigorous protocols, and trial results may not always represent individual smaller non-trial patient populations.

MARK GENOVESE, MD, Stanford University, Rheumatology, 1000 Welch Road \#203, Palo Alto, California 94304, USA. Address correspondence to Dr. M. Genovese; E-mail: genovese@stanford.edu

\section{REFERENCES}

1. Genovese MC, Covarrubias A, Leon G, Mysler E, Keiserman M, Valente R, et al. Subcutaneous abatacept versus intravenous abatacept: a phase IIIb noninferiority study in patients with an inadequate response to methotrexate. Arthritis Rheum 2011;63:2854-64.

2. Genovese MC, Tena CP, Covarrubias A, Leon G, Mysler E, Keiserman M, et al. Subcutaneous abatacept for the treatment of rheumatoid arthritis: longterm data from the ACQUIRE trial. J Rheumatol 2014;41:629-39.

3. Keystone EC, Kremer JM, Russell A, Box J, Abud-Mendoza C, Elizondo MG, et al. Abatacept in subjects who switch from intravenous to subcutaneous therapy: results from the phase IIIb ATTUNE study.Ann Rheum Dis 2012;71:857-61.

4. Reggia R, Franceschini F, Tincani A, Cavazzana I. Switching from intravenous to subcutaneous formulation of abatacept: a single-center Italian experience on efficacy and safety. J Rheumatol 2015;42:193-5.

J Rheumatol 2015;42:10; doi:10.3899/jrheum.150600 